\title{
miR-660 promotes liver cancer cell proliferation by targeting PPP2R2A
}

\author{
YAOZHEN PAN $^{1,2}$, LEI ZHAN $^{3}$, LING CHEN $^{2}$, LIWEN CHEN $^{2}$ and CHENGYI SUN ${ }^{2}$ \\ ${ }^{1}$ Department of Biliary-Hepatic Surgery, The Affiliated Tumor Hospital of Guizhou Medical University, Guiyang, \\ Guizhou 550000; ${ }^{2}$ Department of Biliary-Hepatic Surgery, The Affiliated Hospital of Guizhou Medical University, \\ Guiyang, Guizhou 550025; ${ }^{3}$ Department of Biliary-Hepatic Surgery, The Second Affiliated Hospital of \\ Guizhou University of Traditional Chinese Medicine, Guiyang, Guizhou 550000, P.R China
}

Received October 14, 2018; Accepted November 25, 2019

DOI: $10.3892 /$ etm.2021.10115

\begin{abstract}
Liver cancer (LC) is the leading cause for tumor-related death worldwide, and microRNAs (miRs) have been demonstrated to regulate the progression of LC. In the current study, the function of miR-660 in LC cells was investigated, and the results indicated that miR-660 was highly expressed in LC tissues and cells. This increased expression promoted LC cell proliferation and increased the percentage of S phase cells, while miR-660 knockdown inhibited cell proliferation and increased the percentage of $G_{0} / G_{1}$ phase cells. A Ser/Thr phosphatase protein phosphatase 2 regulatory subunit $\beta \alpha$ (PPP2R2A) was indicated as the target of miR-660, and miR-660 could inhibit PPP2R2A levels. The luciferase reporter assay suggested that miR-660 directly bound to the 3'-untranslated region of PPP2R2A. Additionally, it was revealed that miR-660 inhibited $\mathrm{p} 21$ expression and promoted cyclin D1 expression, confirming that miR-660 regulated LC cell proliferation by regulating cell cycle progression. The double knockdown of miR-660 and PPP2R2A promoted LC cell proliferation, suggesting that miR-660 promoted LC proliferation by targeting PPP2R2A.
\end{abstract}

\section{Introduction}

MicroRNAs (miRNAs or miRs) are small non-coding RNAs that regulate gene expression at the post-transcriptional level and can degrade mRNA or/and inhibit mRNA translation by binding to the 3'-untranslated region (UTR) of target mRNAs. For example, miR-1891b directly binds to the 3'-UTR of cbl proto-oncogene B (Cbl-b) and degrades Cbl-b mRNA (1).

Correspondence to: Dr Chengyi Sun, Department of Biliary-Hepatic Surgery, The Affiliated Hospital of Guizhou Medical University, 28 Guiyi Street, Guiyang, Guizhou 550025, P.R. China

E-mail: chengyisun@medmail.com.cn

Key words: microRNA-660, hepatocellular carcinoma, cell proliferation, protein phosphatase 2 regulatory subunit $\beta \alpha$
Additionally, miRNAs increase mRNA translation or stability by binding to the 5'-UTR of target mRNAs. For example, miR-10a interacts with the 5 '-UTR of ribosomal protein mRNAs to increase their translation (2). miR-483-5p directly binds to the 5'-UTR of insulin like growth factor 2 (IGF2) and increases IGF2 expression (3). miRNAs have also been demonstrated to regulate tumor progression in various types of cancer (4). Liver cancer (LC) is a malignant tumor with a poor prognosis (5). The molecular mechanism of LC progression requires further assessment; however, a number of studies have revealed that miRNAs regulate LC progression. miR-7 has been revealed to inhibit LC growth and metastasis by targeting phosphatidylinositol-4 5-bisphosphate 3-kinase catalytic subunit $\Delta$, mTOR and ribosomal protein S6 kinase $\beta-1$, and regulate the PI3K/Akt pathway (6). miR-124-1 inhibits LC growth by targeting CASC 3 exon junction complex subunit to inactivate the p38/MAPK, JNK and ERK pathways (5).

Protein phosphatase 2 regulatory subunit $\beta \alpha$ (PPP2R2A) is a regulatory subunit of tumor suppressor protein phosphatase 2 (PP2A). PP2A can inhibit PI3K/AKT/mTOR, Wnt/ $\beta$-catenin and MAPK signaling to suppress tumor initiation, growth, invasion and metastasis and induce apoptosis $(7,8)$. PPP2R2A inhibits the progression of a variety of tumors including pancreatic (9), bladder and breast (10) cancer. miR-660 has been demonstrated to be associated with the development of various diseases including graves' disease (11), facioscapulohumeral dystrophy (12) and Alzheimer's disease (13). miR-660 has also been revealed to regulate tumor progression. miR-660 has been demonstrated to be downregulated in lung cancer tissues and patients with low miR-660 exhibited poor outcomes including increased mortality (14). The overexpression of miR-660 has been indicated to inhibit lung cancer cell proliferation, migration and invasion and induce apoptosis. Analysis of the mechanisms associated with miR-660 has revealed that the p53 regulator mouse double minute 2 homolog (MDM2) is a miR-660 target (14). miR-660 suppresses lung cancer progression by inhibiting MDM2 and stabilizing p53 (14). The role of miR-660 in LC progression has not, to the best of our knowledge, been previously assessed. In the present study, the role of miR-660, also known as miR-660-5p, in LC cell proliferation was assessed. miR-660 expression was 
measured in LC cells and tissues, and the effect of miR-660 overexpression and knockdown on LC cell proliferation was assessed. Finally, the regulatory mechanism of miR-660 was investigated.

\section{Materials and methods}

Cell culture. A total of six human LC cell lines (MHCC97H, HCCC-9810, MHCC97L, HepG2, Huh7 and Hep3B) and normal liver cell line THLE-3 (ATCC ${ }^{\circledR}$ CRL-11233 ${ }^{\mathrm{TM}}$ ) were purchased from American Type Culture Collection and cultured according to manufacturer's protocol, briefly, DMEM/high glucose (Hyclone; GE Healthcare Life Sciences) supplemented with 10\% FBS (Hyclone; GE Healthcare Life Sciences) at $37^{\circ} \mathrm{C}$ with $5 \% \mathrm{CO}_{2}$.

Clinical specimens. LC tissues and adjacent normal liver tissues were obtained from The Second Affiliated Hospital of Soochow University (Suzhou, China) between July 2015 and September 2018 according to Edmondson criteria (5). Adjacent normal tissues distanced from tumor tissue above $5 \mathrm{~cm}$. The current study was approved by the Ethics Committee of The Second Affiliated Hospital of Soochow University. Prior patient consent was obtained for the obtainment of specimens (Table S1).

Transfection. The sequence of pre-miR-660 was cloned into a pMSCV-puro vector (Clontech Laboratories, Inc.). An empty and miR-660 overexpressing vector $(10 \mu \mathrm{g})$ were co-transfected with packaging plasmid pIK $(10 \mu \mathrm{g})$ into $293 \mathrm{~T}$ cells (American Type Culture Collection) using a standard calcium phosphate transfection method, as previously described (15). The virus supernatants were collected using $0.45 \mu \mathrm{m}$ filter (EMD Millipore) $36 \mathrm{~h}$ following co-transfection. HepG2 and HepB3 cells were incubated with virus supernatants overnight at $37^{\circ} \mathrm{C}$ with multiplicity of infection $(\mathrm{MOI})=20$. Supernatants were subsequently removed and cells were cultured using DMEM/high glucose (Hyclone; GE Healthcare Life Sciences) with 10\% FBS (Hyclone; GE Healthcare Life Sciences) and $2 \mu \mathrm{g} / \mathrm{ml}$ puromycin (Sigma-Aldrich; Merck) for 1 week to construct the stably miR-660 overexpressing cells. The miR-660 mimic (indicated as miR-660), miR-660 inhibitor (indicated as miR-660-in; cat. no. miR20003338-1-5), miR-660 mimic with seed site mutation (miR-660-mut) and their negative control were synthesized by Guangzhou RiboBio Co., Ltd. and transfected into HepG2 and Hep3B cells using Lipofectamine ${ }^{\circledR} 2000$ to perform luciferase assay (Thermo Fisher Scientific, Inc.) according to manufacturers' protocol. $12 \mathrm{~h}$ after transfection, functional experiments could be performed. The small interfering RNA (siRNA) of PPP2R2A (siPPP2R2A; Guangzhou RiboBio Co., Ltd.) sequences were as follows: PPP2R2AsiRNA\#1, 5'-GAUCCC AGUAACAGGUCAUUU-3' and PPP2R2AsiRNA\#2, 5'-GCA AGUGGCAAGCGAAAGAAA-3'. A total of $10 \mathrm{nM}$ siRNAs were transfected into cells using Lipofectamine 2000.

Reverse transcription-quantitative (RT-q) PCR. Total RNA was isolated using RNAiso Plus (Takara Bio, Inc) in LC cells. For miRNA analysis, cDNA was synthesized using Hiscript Reverse Transcriptase (Vazyme) and a specific stem-loop primer, the sequence of the primer was: 5'-GTCGTATCCAGT GCAGGGTCCGAGGTATTCGCACTGGATACGACCAAC TC-3'. qPCR was subsequently performed using AceQ qPCR SYBR Green Master Mix (Vazyme) and a CFX96 Touch ${ }^{\mathrm{TM}}$ Real-time PCR detection system (Bio-Rad Laboratories, Inc.). miR-660 primer sequences were as follows: Forward, 5'-GCCCGCTACCCATTGCATATCG-3' and reverse, 5'-GTG CAGGGTCCGAGGT-3'. For mRNA analysis, cDNA was synthesized using Hiscript High Fidelity One Step RT-PCR kit (Vazyme) and qPCR was performed using AceQ qPCR SYBR Green Master Mix (Vazyme) and a CFX96 Touch ${ }^{\mathrm{TM}}$ Real-time PCR detection system (Bio-Rad Laboratories, Inc.) by incubation at $95^{\circ} \mathrm{C}$ for $5 \mathrm{~min}$ followed by 40 amplification cycles $\left(10 \mathrm{sec}\right.$ for denaturation at $95^{\circ} \mathrm{C}$ and $30 \mathrm{sec}$ of hybridization and elongation at $60^{\circ} \mathrm{C}$ ). The specific primer sequences for GAPDH, p21 and cyclin D1 (CCND1) are as follows: GAPDH forward, 5'-GGTGGTCTCCTCTGACTTC-3' and reverse, 5'-CTCTTCCTCTTGTGCTCTTG-3'; p21 forward, 5'-TGT CCGTCAGAACCCATGC-3' and reverse, 5'-AAAGTCGAA GTTCCATCGCTC-3'; CCND1 forward, 5'-GCTGCGAAG TGGAAACCATC- 3 ' and reverse, 5'-CCTCCTTCTGCACAC ATTTGAA-3'. U6 was used as an endogenous control for microRNA data normalization, and GAPDH was used as an endogenous control for gene normalization.

Western blot analysis. Total protein of HepG2 was isolated using RIPA buffer [50 mM Tris (pH 7.4); $150 \mathrm{mM} \mathrm{NaCl}$; $1 \%$ NP-40; $0.5 \%$ sodium deoxycholate] supplemented with protease inhibitor cocktail (Roche Applied Science), BCA Protein Assay kit (Thermo Fisher Scientific, Inc.) was used to determine protein concentration, $20 \mu \mathrm{g}$ proteins were separated using $12 \%$ SDS-PAGE, transferred onto PVDF membranes, membranes were blocked using $5 \%$ non-fat milk at room temperature for $2 \mathrm{~h}$, and immunoblotted with the following primary antibodies for overnight at $4{ }^{\circ} \mathrm{C}$ : Anti-p21 (1:1,000; cat. no. ab109520) and anti-CCND1 $(1 ; 1,000$; cat. no. ab137875) antibodies were used (all, Abcam). $\alpha$-Tubulin (1:1,000; cat. no. ab7291; Abcam) was used as the loading control. The secondary antibody (HRP-Goat Anti-Mouse IgG H\&L (1:5,000; cat. no. ab205719; Abcam) and HRP-Goat Anti-Rabbit IgG H\&L (1:5,000; cat. no. ab214880; Abcam) for $1 \mathrm{~h}$ at room temperature. Bands were detected using ECL Western Blotting Detection kit (GE Healthcare).

MTT assay. A total of $3 \times 10^{3} \mathrm{HepG} 2$ cells with miR-660 overexpression were seeded in 96-well plates and stained with $0.05 \mathrm{mg}$ MTT (Sigma-Aldrich; Merck KGaA) at day 0-4 and 5 for $4 \mathrm{~h}$ at $37^{\circ} \mathrm{C}$. DMEM medium supplemented with $10 \% \mathrm{FBS}$ was removed and DMSO was added (Sigma-Aldrich; Merck $\mathrm{KGaA}$ ). Absorbance was measured at a wavelength of $570 \mathrm{~nm}$.

Anchorage-independent growth assay and bromodeoxyuridine $(B r d U)$ incorporation assay. An anchorage-independent growth assay and a BrdU incorporation assay were performed according to a previous method (16).

Cell cycle assay. Cells were trypsined and washed with cold PBS buffer three times and fixed with $70 \%$ cold ethanol at $-20^{\circ} \mathrm{C}$ for $4 \mathrm{~h}$. Cells were spun down and resuspended using PBS, and $2 \mu \mathrm{g} / \mathrm{ml}$ RNAase (Takara Bio, Inc) was added for 
A

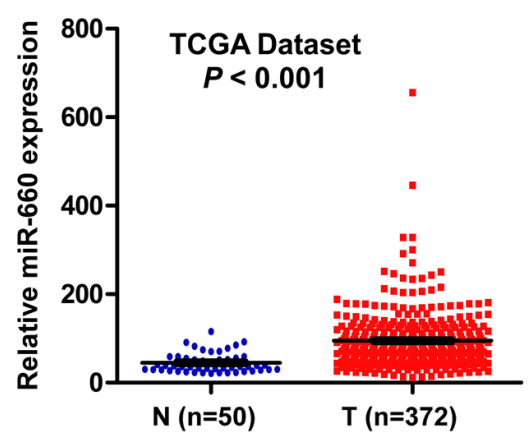

B

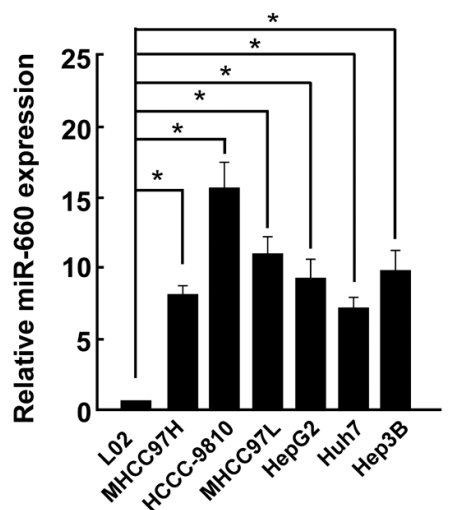

C

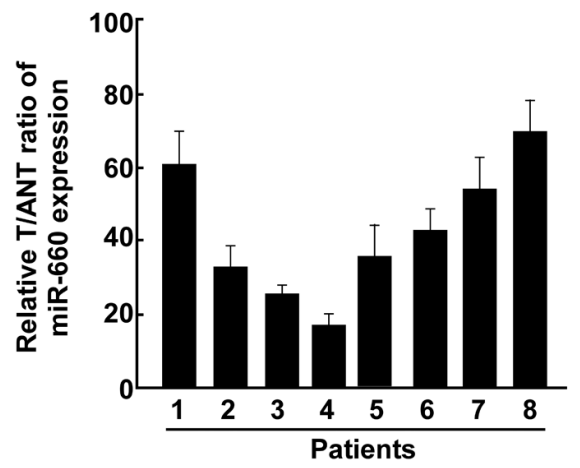

Figure 1. Upregulation of miR-660 in LC tissues. (A) miR-660 expression in LC tissues and normal liver tissues, analyzed using The Cancer Genome Atlas data. (B) miR-660 expression in a normal liver cell line THLE-3 and liver cancer cell lines MHCC97H, HCCC-9810, MHCC97L, HepG2, Huh7 and Hep3B. (C) miR-660 expression in T and ANT. Error bars indicate SEM. "P<0.05. miR, microRNA; LC, liver cancer; T, LC tissues; N, normal liver tissues; ANT, adjacent normal liver tissues.

A

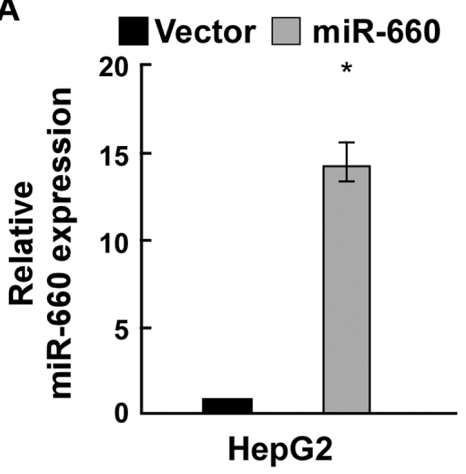

C

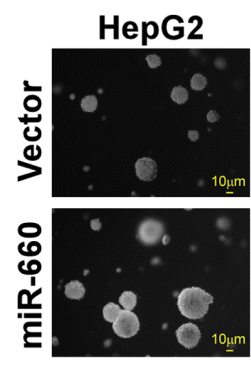

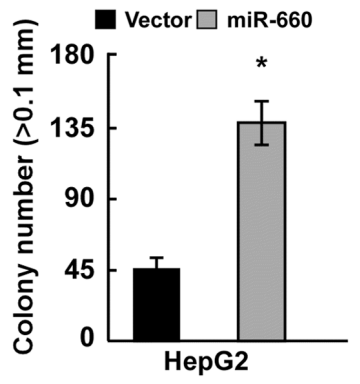

B

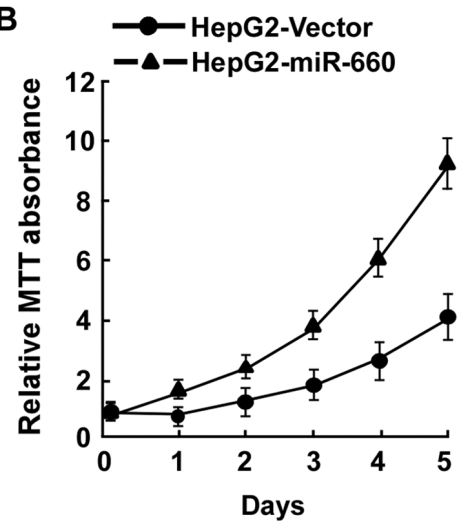

D

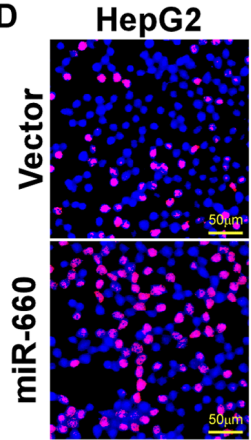

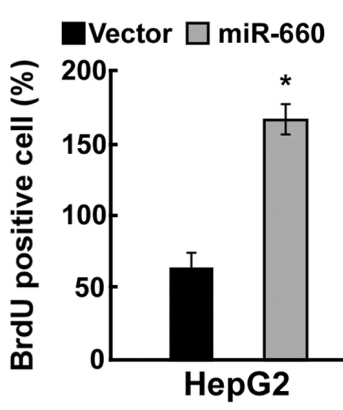

HepG2
E

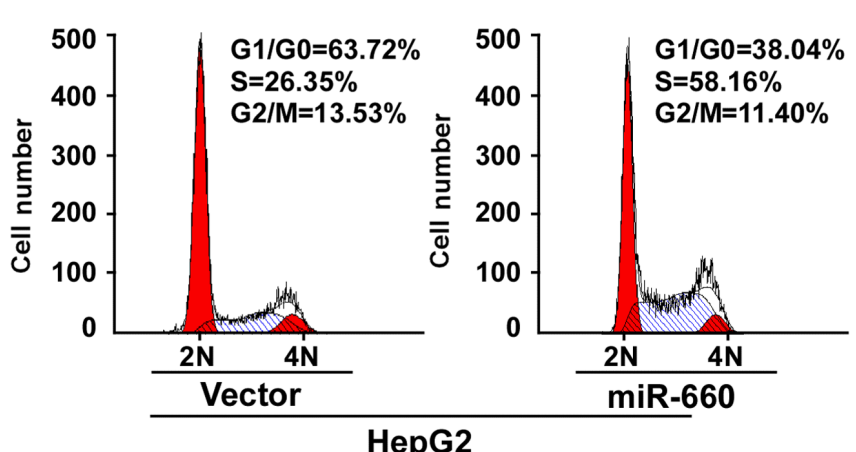

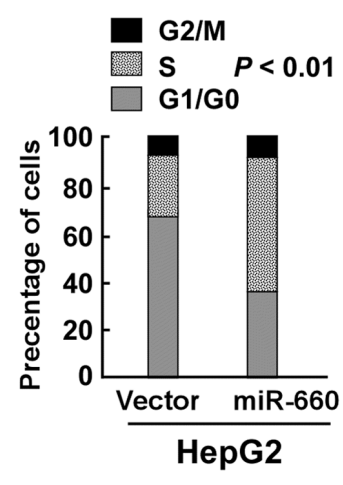

Figure 2. HepG2 proliferation following miR-660 overexpression. (A) Reverse transcription-quantitative PCR analysis of miR-660 expression following transfection using a miR-660 overexpression vector. (B) MTT assay of HepG2 cell proliferation following miR-660 overexpression. (C) Soft agar growth assay of the anchorage-independent growth of HepG2 cells overexpressing miR-660. (D) BrdU incorporation assay of cell proliferation of HepG2 cells overexpressing miR-660. (E) Cell cycle assay of cell cycle distribution in HepG2 cells overexpressing miR-660. Scale bar, $50 \mu \mathrm{m}$. Error bars indicate SEM. "P<0.05. miR, microRNA; BrdU, bromodeoxyuridine. 


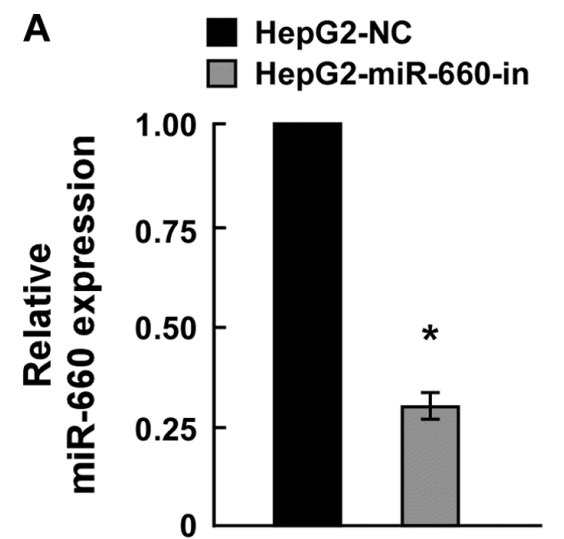

C
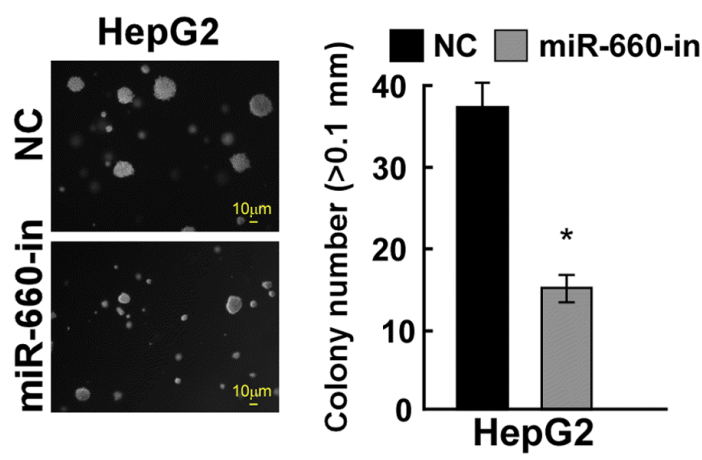

B

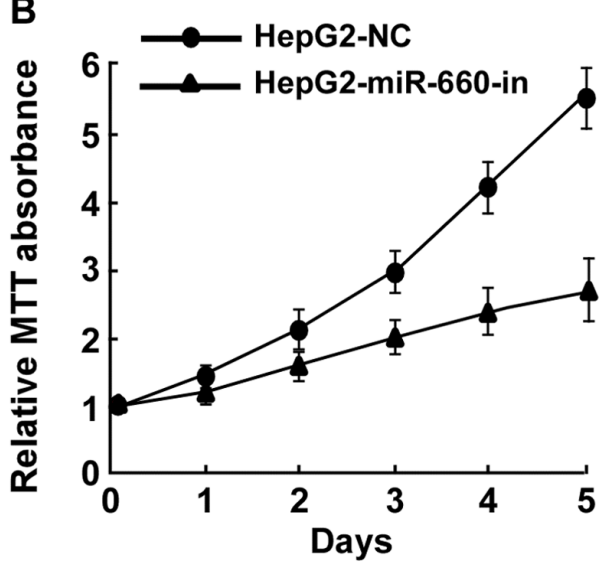

D

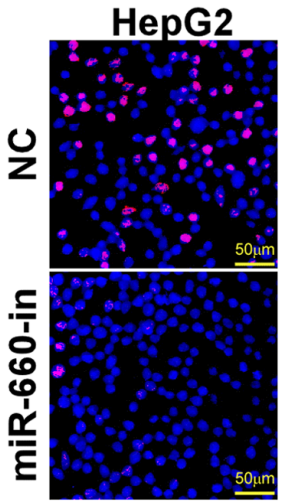

$\square$ NC $\square$ miR-660-in

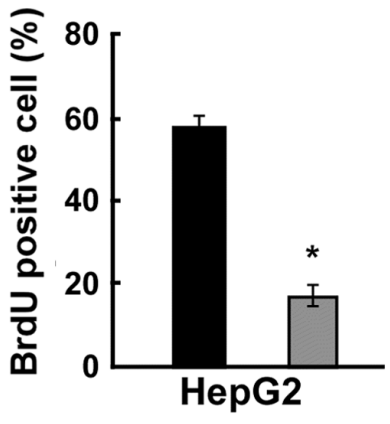

E

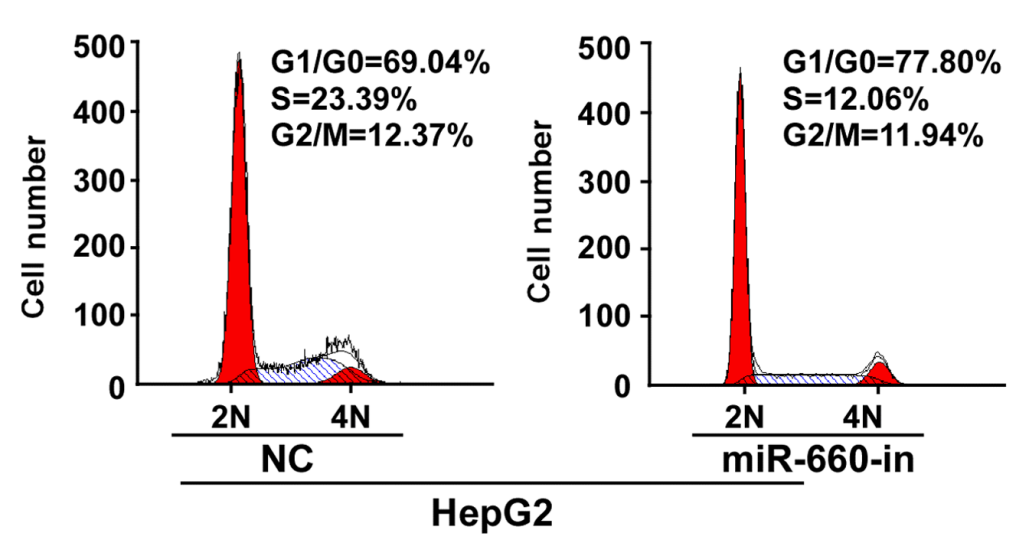

G2/M

S $P<0.01$

G1/G0

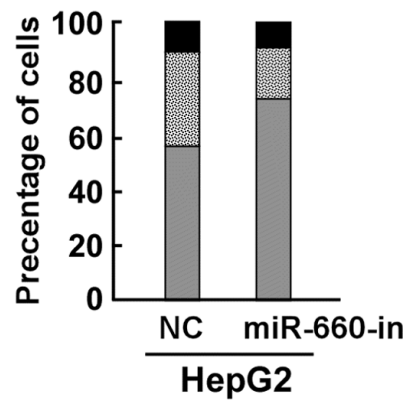

Figure 3. HepG2 proliferation following miR-660 knockdown. (A) Reverse transcription-quantitative PCR analysis of miR-660 expression following transfection using miR-660 overexpression vector. (B) MTT assay of the HepG2 cell proliferation following miR-660 knockdown. (C) Soft agar growth assay of the anchorage-independent growth of HepG2 cells with knocked down miR-660. (D) BrdU incorporation assay of cell proliferation of HepG2 with knocked down miR-660. (E) Cell cycle assay of cell cycle distribution in HepG2 cells with knocked down miR-660. Scale bar, $50 \mu$ for 3C and 3D, Error bars indicate SEM. ${ }^{*} \mathrm{P}<0.05$. miR, microRNA; NC, negative control; in, inhibitor; BrdU, bromodeoxyuridine.

$30 \mathrm{~min}$ at $37^{\circ} \mathrm{C}$, followed by incubation in $20 \mu \mathrm{g} / \mathrm{ml}$ propidium iodide (Sigma-Aldrich; Merck KGaA) at $37^{\circ} \mathrm{C}$ for 30 min. Finally, cells were analyzed using a flow cytometer (FACSCalibur; BD Biosciences).

Luciferase reporter assay. The sequence of 3'-UTR of PPP2R2A containing the binding sites of miR-660 was cloned into a psiCHECK-2 vector (Promega Corporation). Cells were seeded in 24-well plates and co-transfected with psiCHECK2-PPP2R2A-3'-UTR and miR-660 mimic, miR-660 inhibitor or mutational miR-660 mimic (miR-660-mut) into HepG2 using Lipofectamine 2000. Luciferase reporter assay was performed using a Dual-Luciferase Reporter assay kit (Promega Corporation) at $48 \mathrm{~h}$ following co-transfection, according to the manufacturer's protocol. Renilla luciferase was used to normal data.

Statistical analysis. Data analysis was performed using SPSS 20.0 (IBM Corp.), the results are presented as the mean \pm SEM. Comparisons between two groups were performed using an ANOVA. TCGA data was downloaded from www. portal.gdc. cancer.gov. TargetScan 7.2 was used to predict the targets of miR-660. $\mathrm{P}<0.05$ was considered to indicate a statistically significant difference. 
A

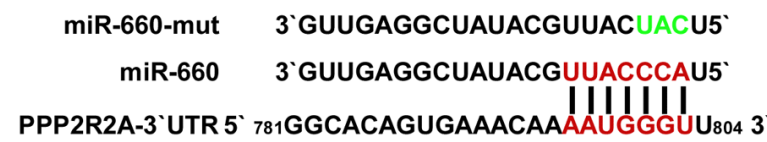

B

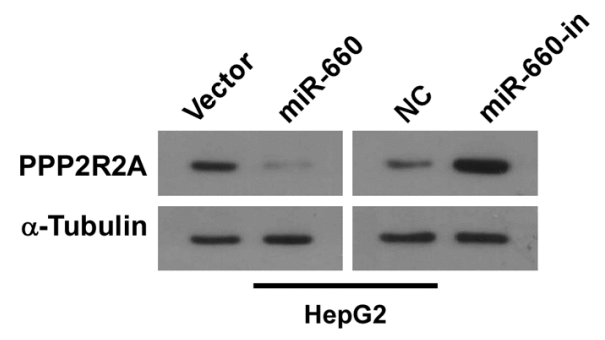

E

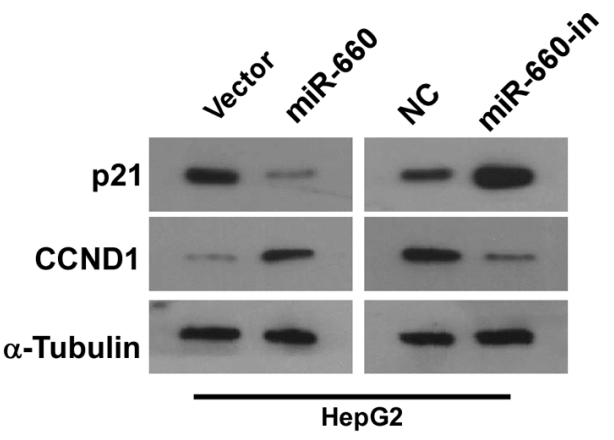

$\mathbf{F}$
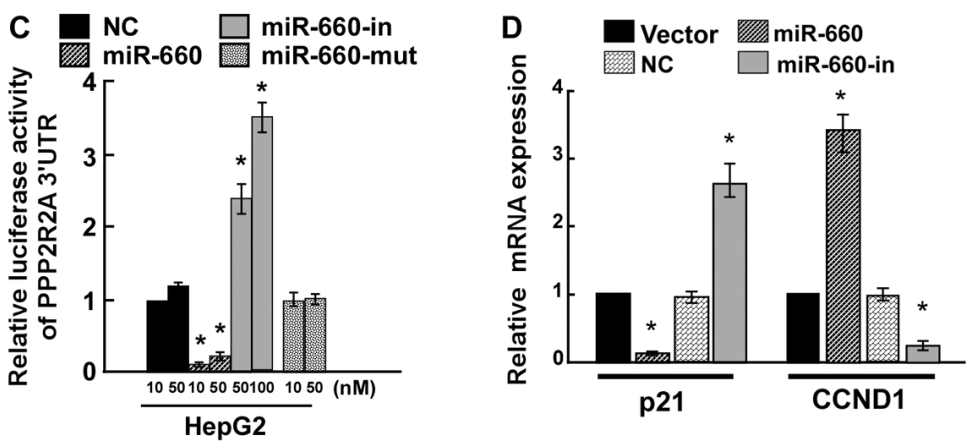

G

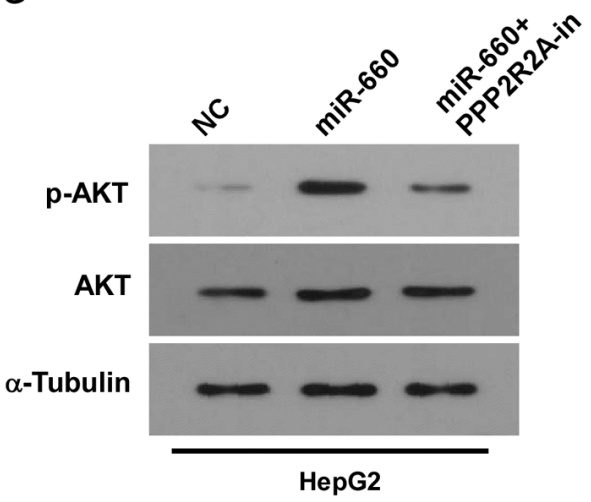

HepG2

Figure 4. PPP2R2A is the target of miR-660. (A) PPP2R2A binding sites with miR-660. Green indicated mutant base, while red indicated wild base. (B) Western blot analysis of PPP2R2A expression in HepG2 cells transfected with miR-660 and miR-660-in. $\alpha$-Tubulin was used as the loading control. (C) Luciferase reporter assay of luciferase activity in HepG2 co-transfected with psiCHECK-2-PPP2R2A-3'-UTR and miR-660 mimic, miR-660-in or miR-660-mut. (D) Reverse transcription-quantitative PCR analysis of p21 and CCND1 expression in HepG2 cells with miR-660 overexpression or knockdown. (E) Western blot analysis of p21 and CCND1 expression in HepG2 with miR-660 overexpression or knockdown. $\alpha$-Tubulin was used as the loading control. (F) Luciferase reporter assay of HepG2 transfected with pGL3-PPP2R2A-3'-UTR or pGL3-PPP2R2A-3'-UTR-mut and increasing amounts of miR-660 or miR-660 inhibitor oligonucleotides. (G) Western blot analysis of the expression of AKT and p-AKT in HepG2 transfected with miR-660 or miR-660 plus PPP2R2A siRNA. Error bars indicate SEM. "P $<0.05$. PPP2R2A, phosphatase protein phosphatase 2 regulatory subunit $\beta \alpha$; miR, microRNA; in, inhibitor; UTR, untranslated region; CCND1, cyclin D1; mut, mutant; si, small interfering.

\section{Results}

miR-660 is upregulated in LC tissues and cells. The miRNA expression profile of LC tissues $(\mathrm{n}=372)$ and normal liver tissues $(\mathrm{n}=50)$ was obtained from The Cancer Genome Atlas dataset and used to analyze miR-660 expression. miR-660 was demonstrated to be significantly upregulated in LC tissues (T) compared with normal liver samples (N) (Fig. 1A). miR-660 expression was also examined in all LC cell lines and a normal liver cell line, and the results revealed that miR-660 was significantly upregulated in LC cells compared with normal liver cells (Fig. 1B). miR-660 expression was further examined in LC tissues and paired adjacent normal liver tissues and the results indicated that miR-660 was upregulated in LC tissues (Fig. 1C), suggesting that miR-660 may promote LC progression.

miR-660 promotes $L C$ cell proliferation. To determine the role of miR-660 in LC cell proliferation, miR-660 was overexpressed in LC cell HepG2 (Fig. 2A). The MTT assay demonstrated that miR-660 overexpression promoted LC cell proliferation (Fig. 2B). Anchorage-independent growth assay revealed that miR-660 overexpression significantly promoted anchorage-independent growth (Fig. 2C). The BrdU incorporation assay indicated that miR-660 overexpression significantly increased the number of BrdU-positive cells, suggesting that miR-660 promoted LC cell proliferation (Fig. 2D). A cell cycle assay was used to confirm the results 
A

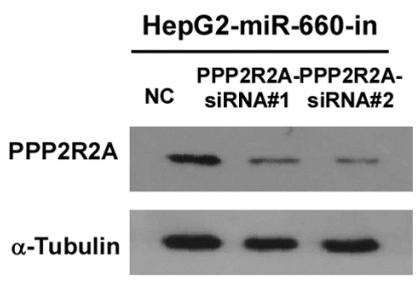

B

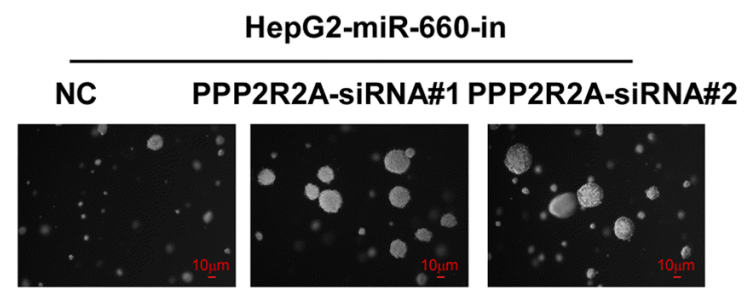

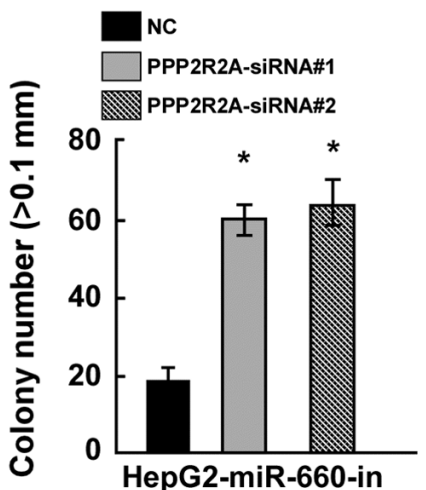

C

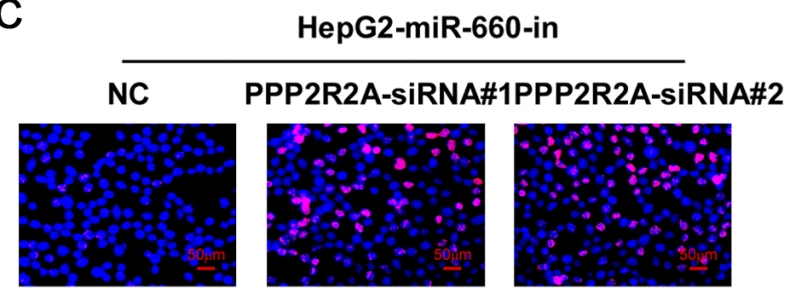

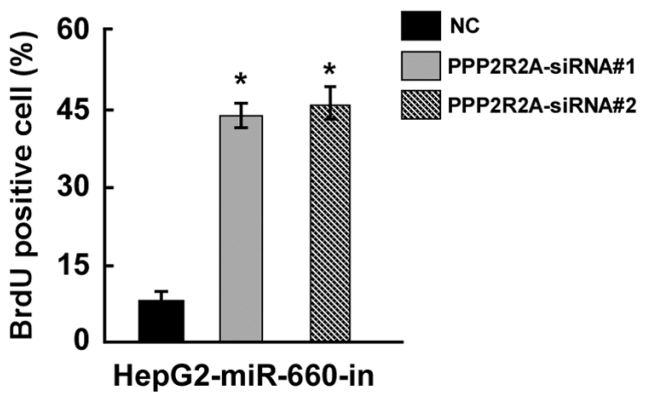

Figure 5. PPP2R2A expression in HepG2 cells transfected with miR-660-in. (A) Western blot analysis of PPP2R2A expression in HepG2 cells co-transfected with miR-660-in and PPP2R2A siRNA. $\alpha$-Tubulin was used as the loading control. (B) Soft agar growth assay of the anchorage-independent growth of HepG2 with knockdown of miR-660 and PPP2R2A. (C) BrdU incorporation assay of HepG2 cell proliferation with knockdown of miR-660 and PPP2R2A. Error bars indicate SEM. * $\mathrm{P}<0.05$ vs. NC. PPP2R2A, phosphatase protein phosphatase 2 regulatory subunit $\beta \alpha$; miR, microRNA; siRNA, small interfering RNA; in, inhibitor; NC, negative control; BrdU, bromodeoxyuridine.

observed. miR-660 overexpression increased the percentage of $\mathrm{S}$ phase cells from 26.35 to $58.16 \%$ and reduced the percentage of $\mathrm{G}_{0} / \mathrm{G}_{1}$ phase cells from 63.72 to $38.04 \%$ (Fig. 2E). These results suggested that miR-660 increased LC cell proliferation through accelerating cell cycle progression.

To confirm these results, miR-660 was knocked down in HepG2 cells (Fig. 3A) The MTT assay demonstrated that miR-660 knockdown inhibited LC cell proliferation (Fig. 3B). A anchorage-independent growth assay indicated that miR-660 knockdown significantly inhibited cell anchorage-independent growth (Fig. 3C). The BrdU incorporation assay demonstrated that miR-660 knockdown significantly reduced the number of BrdU-positive cells (Fig. 3D). The cell cycle assay revealed that miR-660 knockdown increased the percentage of $G_{0} / G_{1}$ phase cells from 69.04 to $77.80 \%$ and reduced the percentage of $\mathrm{S}$ phase cell from 23.39 to $12.06 \%$ (Fig. 3E). These findings suggested that miR-660 promoted LC cell proliferation.

$P P P 2 R 2 A$ is the target of $m i R-660 . \mathrm{miRNAs}$ regulate a variety of biological functions through targeting mRNA. TargetScan 7.2 predicted that PPP2R2A was the target of miR-660 (Fig. 4A). PPP2R2A is a regulatory subunit of the PP2A which is a major Ser/Thr phosphatase (17). Western blot analysis revealed that miR-660 inhibited PPP2R2A expression (Fig. 4B). The results of the luciferase reporter assay indicated that miR-660 overexpression significantly reduced luciferase activity compared with the vector control, miR-660 knockdown significantly increased luciferase activity compared with the negative control, while mutational miR-660 overexpression luciferase activity did not change (Fig. 4C). These results suggested that miR-660 directly bound to the 3'-UTR of PPP2R2A and PPP2R2A was the target of miR-660. p21 and CCND1 are important cell cycle regulators and have been revealed to regulate cell proliferation $(18,19)$. The results of the current study indicated that miR-660 overexpression significantly increased CCND1 expression and decreased p21 mRNA and protein expression. miR-660 knockdown markedly inhibited CCND1 expression and increased p21 expression (Fig. 4D and E). These findings further confirmed that miR-660 promoted HepG2 proliferation by targeting PPP2R2A.

miR-660 promotes LC cell proliferation through inhibiting PPP2R2A. To confirm whether miR-660 promoted LC cell proliferation by targeting PPP2R2A, miR-660 and PPP2R2A were knocked down in HepG2 cells and the results of western blot analysis indicated that PPP2R2A siRNAs inhibited PPP2R2A expression (Fig. 5A). The colony formation assay suggested that miR-660 and PPP2R2A knockdown significantly promoted HepG2 proliferation compared with miR-660 knockdown alone (Fig. 5B). The BrdU incorporation assay demonstrated that miR-660 and PPP2R2A knockdown increased the number of BrdU positive cells compared with miR-660 knockdown alone (Fig. 5C). These findings suggested that miR-660 promoted LC cell proliferation through the inhibition of PPP2R2A.

\section{Discussion}

The role of miRNAs in liver cancer progression has been extensively studied. For example, miRNA-503 has been 
indicated to inhibit the proliferation of liver cancer by targeting cyclin D3 and E2F transcription factor 1 (20). miR-122 has also been revealed to inhibit liver cancer growth by targeting MDM2 (21). Numerous miRNAs have been indicated to serve a role in the progression of liver cancer by targeting a variety of the same or different genes. In the present study, miR-660 was revealed to be upregulated in LC cells and tissues, and cellular function analysis revealed that miR-660 promoted HepG2 proliferation. miR-660 has been previously revealed to be downregulated in lung cancer cells and tissues, and to inhibit lung tumorigenesis, while it promotes liver cancer progression. This may be due to the fact miR-660 targets oncogenes in lung cancer. MDM2 is a target of miR-660 in lung cancer cells and inhibits p53 activity $(14,22)$. In the current study, PPP2R2A was revealed to be the target of miR-660 in liver cancer. Mechanism analysis suggested that PPP2R2A was its target, and the double knockdown of miR-660 and PPP2R2A promoted HepG 2 proliferation, confirming that miR-660 promoted HepG2 proliferation by targeting PPP2R2A.

A number of miRNAs have been demonstrated to regulate PPP2R2A expression, including miR-556-5p (23), miR-136, miR-31 (24), miR-892a (25) and miR-222 (26). These miRNAs promote tumor progression by inhibiting PPP2R2A, further confirming PPP2R2A as a tumor suppressor (26). miR-222 is overexpressed in LC, and promotes LC cell motility by targeting PPP2R2A (27). In the current study, miR-660 promoted LC cell proliferation by targeting PPP2R2A, suggesting that miR-660 may also promote LC metastasis. These results indicate that miR-660 may be a novel target for LC therapy. However, studies using animal models are required to support these findings.

p21 inhibits cell cycle progression and CCND1 promotes cell cycle progression $(18,19)$. In the current study, it was demonstrated that miR-660 inhibited p21 expression and upregulated CCND1 expression, which was consistent with results of the cell cycle assay which indicated that miR-660 promoted cell cycle progression. These results revealed that miR-660 promoted HepG2 proliferation by inhibiting PPP2R2A. In conclusion, miR-660 was upregulated in LC cells and tissues and contributed to $\mathrm{LC}$ cell proliferation by inhibiting PPP2R2A.

\section{Acknowledgements}

Not applicable.

\section{Funding}

No funding was received.

\section{Availability of data and materials}

All data generated or analyzed during this study are included in this published article.

\section{Authors' contributions}

CYS and YZP designed the study; YZP, LZ, LC and LWC acquired the data; CYS and YZP wrote and revised the manuscript. All authors read and approved the final manuscript.

\section{Ethics approval and consent to participate}

The current study was approved by the Ethics Committee of The Second Affiliated Hospital of Soochow University. Prior patient consent was obtained for the obtainment of specimens.

\section{Patient consent of publication}

Not applicable.

\section{Competing interests}

The authors declare that they have no competing interests.

\section{References}

1. Dong Q, Li C, Che X, Qu J, Fan Y, Li X, Li Y, Wang Q, Liu Y, Yang X and Qu X: MicroRNA-891b is an independent prognostic factor of pancreatic cancer by targeting Cbl-b to suppress the growth of pancreatic cancer cells. Oncotarget 7: 82338-82353, 2016.

2. Ørom UA, Nielsen FC and Lund AH: MicroRNA-10a binds the 5'UTR of ribosomal protein mRNAs and enhances their translation. Mol Cell 30: 460-471, 2008.

3. Liu M, Roth A, Yu M, Morris R, Bersani F, Rivera MN, Lu J, Shioda T, Vasudevan S, Ramaswamy S, et al: The IGF2 intronic miR-483 selectively enhances transcription from IGF2 fetal promoters and enhances tumorigenesis. Genes Dev 27: 2543-2548, 2013.

4. Lin Q, Ma L, Liu Z, Yang Z, Wang J, Liu J and Jiang G: Targeting microRNAs: A new action mechanism of natural compounds. Oncotarget 8: 15961-15970, 2017.

5. Xu L, Dai W, Li J, He L, Wang F, Xia Y, Chen K, Li S, Liu T, Lu J, et al: Methylation-regulated miR-124-1 suppresses tumorigenesis in hepatocellular carcinoma by targeting CASC3. Oncotarget 7: 26027-26041, 2016.

6. Fang Y, Xue JL, Shen Q, Chen J and Tian L: MicroRNA-7 inhibits tumor growth and metastasis by targeting the phosphoinositide 3-kinase/Akt pathway in hepatocellular carcinoma. Hepatology 55: 1852-1862, 2012.

7. Liu CC, Lin SP, Hsu HS, Yang SH, Lin CH, Yang MH, Hung MC and Hung SC: Suspension survival mediated by PP2A-STAT3-Col XVII determines tumour initiation and metastasis in cancer stem cells. Nat Commun 7: 11798, 2016.

8. Cristóbal I, Manso R, Rincón R, Caramés C, Senin C, Borrero A, Martínez-Useros J, Rodriguez M, Zazo S, Aguilera O, et al: PP2A inhibition is a common event in colorectal cancer and its restoration using FTY720 shows promising therapeutic potential. Mol Cancer Ther 13: 938-947, 2014.

9. Wang Q, Li J, Wu W, Shen R, Jiang H, Qian Y, Tang Y, Bai T, Wu S, Wei L, et al: Smad4-dependent suppressor pituitary homeobox 2 promotes PPP2R2A-mediated inhibition of Akt pathway in pancreatic cancer. Oncotarget 7: 11208-11222, 2016.

10. Li X, Zhang J and Ma D: PPP2R2A binds and dephosphorylates GFPT2 in breast cancer cells. Sheng Wu Gong Cheng Xue Bao 34: 956-963, 2018 (In Chinese).

11. Qin Q, Wang X, Yan N, Song RH, Cai TT, Zhang W, Guan LJ, Muhali FS and Zhang JA: Aberrant expression of miRNA and mRNAs in lesioned tissues of Graves' disease. Cell Physiol Biochem 35: 1934-1942, 2015.

12. Dmitriev P, Stankevicins L, Ansseau E, Petrov A, Barat A, Dessen P, Robert T, Turki A, Lazar V, Labourer E, et al: Defective regulation of microRNA target genes in myoblasts from facioscapulohumeral dystrophy patients. J Biol Chem 288: 34989-35002, 2013.

13. Satoh J, Kino Y and Niida S: MicroRNA-seq data analysis pipeline to identify blood biomarkers for Alzheimer's disease from public data. Biomarker insights 10: 21-31, 2015.

14. Fortunato O, Boeri M, Moro M, Verri C, Mensah M, Conte D, Caleca L, Roz L, Pastorino U and Sozzi G: Mir-660 is downregulated in lung cancer patients and its replacement inhibits lung tumorigenesis by targeting MDM2-p53 interaction. Cell Death Dis 5: e1564, 2014. 
15. Cribbs AP, Kennedy A, Gregory B and Brennan FM: Simplified production and concentration of lentiviral vectors to achieve high transduction in primary human T cells. BMC Biotechnol 13: 98, 2013.

16. Lin H, Dai T, Xiong H, Zhao X, Chen X, Yu C, Li J, Wang X and Song L: Unregulated miR-96 induces cell proliferation in human breast cancer by downregulating transcriptional factor FOXO3a. PLoS One 5: e15797, 2010.

17. Alvarez-Fernández M, Halim VA, Aprelia M, Laoukili J, Mohammed S and Medema RH: Protein phosphatase 2A (B55 $\alpha$ ) prevents premature activation of forkhead transcription factor FoxM1 by antagonizing cyclin A/cyclin-dependent kinase-mediated phosphorylation. J Biol Chem 286: 33029-33036, 2011.

18. Forte D, Salvestrini V, Corradi G, Rossi L, Catani L, Lemoli RM, Cavo M and Curti A: The tissue inhibitor of metalloproteinases-1 (TIMP-1) promotes survival and migration of acute myeloid leukemia cells through CD63/PI3K/Akt/p21 signaling. Oncotarget 8: 2261-2274, 2017.

19. Pestell RG: New roles of cyclin D1. Am J Pathol 183: 3-9, 2013.

20. Xiao F, Zhang W, Chen L, Chen F, Xie H, Xing C, Yu X, Ding S, Chen K, Guo H, et al: MicroRNA-503 inhibits the G1/S transition by downregulating cyclin D3 and E2F3 in hepatocellular carcinoma. J Transl Med 11: 195, 2013.

21. Simerzin A, Zorde-Khvalevsky E, Rivkin M, Adar R, Zucman-Rossi J, Couchy G, Roskams T, Govaere O, Oren M, Giladi $\mathrm{H}$ and Galun E: The liver-specific microRNA-122*, the complementary strand of microRNA-122, acts as a tumor suppressor by modulating the p53/mouse double minute 2 homolog circuitry. Hepatology 64: 1623-1636, 2016.
22. Fei $\mathrm{L}$ and $\mathrm{Xu} \mathrm{H}$ : Role of MCM2-7 protein phosphorylation in human cancer cells. Cell Biosci 8: 43, 2018.

23. Zhao W, Cao L, Zeng S, Qin H and Men T: Upregulation of miR-556-5p promoted prostate cancer cell proliferation by suppressing PPP2R2A expression. Biomed Pharmacother 75: 142-147, 2015.

24. Shen S, Yue H, Li Y, Qin J, Li K, Liu Y and Wang J: Upregulation of miR-136 in human non-small cell lung cancer cells promotes Erk1/2 activation by targeting PPP2R2A. Tumour Biol 35: 631-640, 2014.

25. Liang WL, Cao J, Xu B, Yang P, Shen F, Sun Z, Li WL, Wang Q and Liu F: miR-892a regulated PPP2R2A expression and promoted cell proliferation of human colorectal cancer cells. Biomed Pharmacother 72: 119-124, 2015.

26. Zeng LP, Hu ZM, Li K and Xia K: miR-222 attenuates cisplatin-induced cell death by targeting the PPP2R2A/Akt/mTOR Axis in bladder cancer cells. J Cell Mol Med 20: 559-567, 2016.

27. Wong QW, Ching AK, Chan AW, Choy KW, To KF, Lai PB and Wong N: MiR-222 overexpression confers cell migratory advantages in hepatocellular carcinoma through enhancing AKT signaling. Clin Cancer Res 16: 867-875, 2010. 